\title{
Wnt signaling in cancer: therapeutic targeting of Wnt signaling beyond $\beta$-catenin and the destruction complex
}

\author{
Youn-Sang Jung ${ }^{1}$ and Jae-l| Park (iD) $1,2,3$
}

\begin{abstract}
Wnt/ $\beta$-catenin signaling is implicated in many physiological processes, including development, tissue homeostasis, and tissue regeneration. In human cancers, Wnt/ $\beta$-catenin signaling is highly activated, which has led to the development of various Wnt signaling inhibitors for cancer therapies. Nonetheless, the blockade of Wnt signaling causes side effects such as impairment of tissue homeostasis and regeneration. Recently, several studies have identified cancer-specific Wnt signaling regulators. In this review, we discuss the Wnt inhibitors currently being used in clinical trials and suggest how additional cancer-specific regulators could be utilized to treat Wnt signaling-associated cancer.
\end{abstract}

\section{Introduction}

Wnt signaling orchestrates various biological processes, such as cell proliferation, differentiation, organogenesis, tissue regeneration, and tumorigenesis ${ }^{1-5}$. Classically, Wnt signaling is divided into $\beta$-catenin-dependent (canonical, Wnt/ $\beta$-catenin pathway) and $\beta$-catenin-independent (noncanonical, Wnt/planar cell polarity $[\mathrm{PCP}]$ and calcium pathway) signaling ${ }^{6,7}$. Canonical Wnt signaling mainly regulates cell proliferation, and noncanonical Wnt signaling controls cell polarity and movement. However, this terminological distinction is unclear, and has been questions by studies proposing the involvement of both $\beta$-catenin-dependent and $\beta$-cateninindependent Wnt signaling in tumorigenesis ${ }^{8}$. For instance, APC and $\beta$-catenin are not only involved in cell proliferation but have also been linked to cell-to-cell adhesion ${ }^{9}$. In this review, we will discuss an ongoing effort to inhibit Wnt signaling and suggest potential approaches

\footnotetext{
Correspondence: Jae-II Park (jaeil@mdanderson.org)

${ }^{1}$ Department of Experimental Radiation Oncology, Division of Radiation

Oncology, The University of Texas MD Anderson Cancer Center, Houston, TX 77030, USA

${ }^{2}$ Graduate School of Biomedical Sciences, The University of Texas MD

Anderson Cancer Center, Houston, TX 77030, USA
}

Full list of author information is available at the end of the article. to target Wnt signaling for cancer therapies proposed from recent studies.

\section{Wnt signaling and clinical trials in human cancers}

$\beta$-Catenin is a crucial signaling transducer in Wnt signaling ${ }^{10,11}$. The $\beta$-catenin protein destruction complex composed of adenomatous polyposis coli (APC), casein kinase 1 (CK1), glycogen synthase kinase $3 \alpha / \beta$ $($ GSK- $3 \alpha / \beta)$, and AXIN1 tightly controls $\beta$-catenin via phosphorylation-mediated proteolysis ${ }^{10,12-16}$. In this section, we briefly describe how genetic alterations of Wnt signaling contribute to tumorigenesis and introduce recent clinical trials that have aimed to inhibit Wnt signaling for cancer treatment.

\section{The $\beta$-catenin destruction complex}

Colorectal cancer (CRC) is the representative of human cancer caused by Wnt signaling hyperactivation ${ }^{17,18}$. CRC displays a high mutation frequency in $A P C(\sim 70 \%)^{19-21}$. In 1991, APC mutation was identified as the cause of hereditary colon cancer syndrome, also called familial adenomatous polyposis ${ }^{22}$. APC forms the $\beta$-catenin destruction complex in association with CK1, AXIN1, and GSK-3 and interacts with $\beta$-catenin ${ }^{15,23,24}$. This protein destruction 
complex downregulates $\beta$-catenin through phosphorylation and ubiquitin-mediated protein degradation ${ }^{10,12-16}$. Genetic mutations causing the loss of function of the destruction complex or gain of function of $\beta$-catenin lead to nuclear translocation of $\beta$-catenin, resulting in T-cell factor (TCF)4/ $\beta$-catenin-mediated transactivation of Wnt target genes ${ }^{25,26}$. The Vogelstein group established a multistep tumorigenesis model of CRC. APC mutation is an early event that initiates CRC adenoma ${ }^{27}$. CRC progression also requires additional genetic alterations in KRAS, PI3K, TGF- $\beta, S M A D 4$, and $T P 53^{27}$. Moreover, epigenetic silencing of negative regulators of Wnt signaling was also frequently found in the absence of $A P C$ mutations ${ }^{28,29}$. APC is a multifunctional protein. In addition to its role in $\beta$-catenin degradation, APC binds to actin and actin-regulating proteins ${ }^{30-33}$, which controls the interaction between E-cadherin and $\alpha-/$ $\beta$-catenin and various physiological processes, including migration and chromosomal fidelity ${ }^{34-38}$. Importantly, recent studies revealed that $A P C$ mutation is insufficient to fully activate Wnt signaling. Furthermore, even if $A P C$ is mutated, mutant APC still negatively regulates $\beta$-catenin to some extent ${ }^{39,40}$, which will be discussed later.

AXIN1 is a multidomain scaffolding protein that forms the $\beta$-catenin destruction complex in association with $\mathrm{APC}, \mathrm{CK} 1$, and GSK $3^{10,41,42}$. In human cancer, AXIN1 mutations are scattered throughout the whole coding sequence of the AXIN1 gene ${ }^{43,44}$, which results in disassembly of the $\beta$-catenin destruction complex. As a priming kinase, CK1 initially phosphorylates $\beta$-catenin (Ser45), which induces the sequential phosphorylation of $\beta$-catenin by GSK3. Subsequently, phosphorylated $\beta$-catenin is recognized and degraded by E3 ubiquitin ligase $(\beta-\operatorname{TrCP})^{10,12-16}$. GSK3 is a serine/threonine kinase that phosphorylates three serine/threonine residues of $\beta$-catenin (Ser33, Ser37, and Thr41) ${ }^{45,46}$. Since GSK3 does not bind to $\beta$-catenin directly, AXIN1 and APC facilitate the interaction of GSK3 with $\beta$-catenin ${ }^{47,48}$. Moreover, unphosphorylated AXIN1 shows a low binding affinity to $\beta$-catenin, which is increased by phosphorylation of AXIN1 via GSK3 kinase activity ${ }^{49,50}$. Low-density lipoprotein receptor-related protein 5/6 (LRP5/6) coreceptor is also phosphorylated by CK1 and GSK3, leading to the recruitment of AXIN1 to the membrane ${ }^{51-53}$.

\section{WNT ligands and receptors}

Under physiological conditions, Wnt signaling is activated by the binding of secreted WNT ligands to LRP5/6 coreceptors and frizzled (FZD) receptors ${ }^{54}$, which induces the recruitment of the protein destruction complex to the LRP receptors and the subsequent phosphorylation of the Ser/Pro-rich motif of the LRP cytoplasmic domain via GSK3 ${ }^{15,55,56}$. This event activates dishevelled (DVL) and inhibits GSK3, resulting in the inhibition of the phosphorylation-mediated $\beta$-catenin protein degradation and the stabilization/accumulation of $\beta$-catenin. Then, $\beta$-catenin undergoes nuclear translocation and transactivates Wnt target genes ${ }^{57}$. The secretion of WNT ligands mainly depends on acylation by Porcupine (PORCN $)^{58,59}$. PORCN is a membrane-bound O-acyltransferase that mediates the palmitoylation of WNT ligands to induce their secretion. In line with this observation, PORCN shows increased genetic alterations in various human cancers, including esophageal, ovarian, uterine, lung, and cervical cancers ${ }^{60}$.

\section{Mutations in CTNNB1/ $\beta$-catenin}

Unlike CRC, in which the $A P C$ gene is frequently mutated, the CTNNB1 gene encoding $\beta$-catenin is predominantly mutated in hepatocellular carcinoma, endometrial cancer, and pancreatic cancer ${ }^{61-63}$. The CTNNB1/ $\beta$-catenin gene harbors 16 exons. $\beta$-Catenin is mainly composed of three domains (N-terminal [ 150 aa], armadillo repeat [12 copies; $550 \mathrm{aa}]$, and C-terminal $[\sim 100 \mathrm{aa}])$. The $\mathrm{N}$-terminal domain contains the phosphorylation sites for GSK3 and CK1 $1^{12,14,45,46}$, which induces $\beta$-TrcP-mediated $\beta$-catenin degradation. The $\mathrm{C}$ terminal domain is involved in transactivation of $\mathrm{Wnt}$ target genes via TCF/LEF interactions ${ }^{25,64-66}$. The armadillo repeat domain interacts with various proteins, including E-cadherin, APC, AXIN1, and PYGOs/Pygopus $^{67,68}$. In human cancer, the phosphorylation sites (Ser/ $\mathrm{Thr}$ ) in the $\mathrm{N}$-terminal domain of $C T N N B 1 / \beta$-catenin are mutational hotspots ${ }^{14,69,70}$, demonstrating that escape from destruction complex-mediated $\beta$-catenin protein degradation is a key process for Wnt signaling-induced tumorigenesis.

\section{Therapeutic targeting of Wnt/ $\mathbf{\beta}$-catenin signaling}

To suppress WNT ligands or receptors for cancer treatment, PORCN inhibitors, WNT ligand antagonists, and FZD antagonists/monoclonal antibodies have been examined in clinical trials of various Wnt signalingassociated human cancers (Table 1 and Fig. 1).

\section{(i) PORCN inhibitors}

WNT974 (LGK974; NIH clinical trial numbers [clinicaltrials.gov]: NCT02278133, NCT01351103, and NCT02649530), ETC-1922159 (ETC-159; NCT02521844), RXC004 (NCT03447470), and CGX1321 (NCT02675946 and NCT03507998) are orally administered PORCN inhibitors that commonly bind to PORCN in the endoplasmic reticulum $^{71-74}$. Therefore, PORCN inhibitors block the secretion of WNT ligands through inhibition of posttranslational acylation of WNT ligands. However, similar to other cancer therapies targeting the Wnt pathway, skeletal side effects such as impairment of bone mass and strength and increase in bone resorption were caused by PORCN inhibitor administration ${ }^{75}$. 
Table 1 Wnt/ $\beta$-catenin signaling inhibitors in current and past clinical trials.

\begin{tabular}{|c|c|c|c|c|}
\hline Drug & Mechanism of action & Cancer type & Phase & Identifier \\
\hline *WNT974 (with LGX818 and Cetuximab) & PORCN inhibitor & Metastatic CRC & Phase 1 & NCT02278133 \\
\hline WNT974 & PORCN inhibitor & $\begin{array}{l}\text { Squamous cell cancer } \\
\text { Head\&Neck }\end{array}$ & Phase 2 & NCT02649530 \\
\hline WNT974 & PORCN inhibitor & $\begin{array}{l}\text { Pancreatic cancer } \\
\text { BRAF mutant CRC } \\
\text { Melanoma } \\
\text { TNBC } \\
\text { H\&N } \\
\text { Squamous cell cancer (cervical, } \\
\text { esophageal, lung) }\end{array}$ & Phase 1 & NCT01351103 \\
\hline ETC-1922159 & PORCN inhibitor & Solid tumor & Phase 1 & NCT02521844 \\
\hline RXC004 & PORCN inhibitor & Solid tumor & Phase 1 & NCT03447470 \\
\hline CGX1321 & PORCN inhibitor & $\begin{array}{l}\text { Colorectal adenocarcinoma } \\
\text { Gastric adenocarcinoma } \\
\text { Pancreatic adenocarcinoma } \\
\text { Bile duct carcinoma } \\
\text { HCC } \\
\text { Esophageal carcinoma } \\
\text { Gastrointestinal cancer }\end{array}$ & Phase 1 & NCT03507998 \\
\hline${ }^{*}$ CGX1321 (with Pembrolizumab) & PORCN inhibitor & $\begin{array}{l}\text { Solid tumors } \\
\text { Gl cancer }\end{array}$ & Phase 1 & NCT02675946 \\
\hline OTSA101-DTPA-90Y & FZD10 antagonist & Sarcoma, Synovial & Phase 1 & NCT01469975 \\
\hline *OMP-18R5 (with Docetaxel) & $\begin{array}{l}\text { Monoclonal antibody against FZD } \\
\text { receptors }\end{array}$ & Solid tumors & Phase 1 & NCT01957007 \\
\hline OMP-18R5 & $\begin{array}{l}\text { Monoclonal antibody against FZD } \\
\text { receptors }\end{array}$ & Metastatic breast cancer & Phase 1 & NCT01973309 \\
\hline OMP-18R5 & $\begin{array}{l}\text { Monoclonal antibody against FZD } \\
\text { receptors }\end{array}$ & Solid tumors & Phase 1 & NCT01345201 \\
\hline $\begin{array}{l}\text { *OMP-18R5 (with Nab-Paclitaxel and } \\
\text { Gemcitabine) }\end{array}$ & $\begin{array}{l}\text { Monoclonal antibody against FZD } \\
\text { receptors }\end{array}$ & $\begin{array}{l}\text { Pancreatic cancer } \\
\text { Stage IV pancreatic cancer }\end{array}$ & Phase 1 & NCT02005315 \\
\hline *OMP-54F28 (with Sorafenib) & FZD8 decoy receptor & $\begin{array}{l}\text { Hepatocellular cancer } \\
\text { Liver cancer }\end{array}$ & Phase 1 & NCT02069145 \\
\hline *OMP-54F28 (with Paclitaxel \& Carboplatin) & FZD8 decoy receptor & Ovarian cancer & Phase 1 & NCT02092363 \\
\hline $\begin{array}{l}\text { *OMP-54F28 (with Nab-Paclitaxel and } \\
\text { Gemcitabine) }\end{array}$ & FZD8 decoy receptor & $\begin{array}{l}\text { Pancreatic cancer } \\
\text { Stage IV pancreatic cancer }\end{array}$ & Phase 1 & NCT02050178 \\
\hline OMP-54F28 & FZD8 decoy receptor & Solid tumors & Phase 1 & NCT01608867 \\
\hline PRI-724 & $\mathrm{CBP} / \beta$-catenin antagonist & $\begin{array}{l}\text { Advanced pancreatic cancer } \\
\text { Metastatic pancreatic cancer } \\
\text { Pancreatic adenocarcinoma }\end{array}$ & Phase 1 & NCT01764477 \\
\hline PRI-724 & $\mathrm{CBP} / \beta$-catenin antagonist & Advanced solid tumors & Phase 1 & NCT01302405 \\
\hline PRI-724 & $\mathrm{CBP} / \beta$-catenin antagonist & $\begin{array}{l}\text { Acute myeloid leukemia } \\
\text { Chronic myeloid leukemia }\end{array}$ & Phase 2 & NCT01606579 \\
\hline $\begin{array}{l}\text { *PRI-724 (with Leucovorin Calcium, Oxaliplatin, } \\
\text { or Fluorouracil) }\end{array}$ & $\mathrm{CBP} / \beta$-catenin antagonist & $\begin{array}{l}\text { Acute myeloid leukemia } \\
\text { Chronic myeloid leukemia }\end{array}$ & Phase 2 & NCT02413853 \\
\hline SM08502 & $\begin{array}{l}\beta \text {-catenin-controlled gene } \\
\text { expression inhibitor }\end{array}$ & Solid tumors & Phase 1 & NCT03355066 \\
\hline
\end{tabular}

(ii) SFRP and SFRP peptides

SFRPs (secreted frizzled-related proteins) are soluble proteins. Given the structural homology of SFRPs with the WNT ligand-binding domain in the FZD receptors, SFRPs function as antagonists that bind to WNT ligands and prevent Wnt signaling activation ${ }^{76-78}$. Indeed, SFRPs or SFRP-derived peptides showed tumor suppressive activity in preclinical models ${ }^{79,80}$.

(iii) FZD antagonist/monoclonal antibody

Vantictumab (OMP-18R5; NIH clinical trial numbers [clinicaltrials.gov]; NCT02005315, NCT01973309, NCT01345201, and NCT01957007) is a monoclonal antibody directly binding to FZD receptors, which blocks the binding of WNT ligands to FZD 1, 2, 5, 7, and $8^{81}$. Ipafricept (OMP-54F28; NIH clinical trial numbers: NCT02069145, NCT02050178, NCT02092363, and NCT01608867) is a recombinant fusion protein that binds to a human IgG1 Fc fragment of FZD $8^{82,83}$. These reagents negatively regulate $\mathrm{Wnt} / \beta$-catenin signaling through their direct binding to FZD, which thereby disrupts the function of LRPs/FZDs. Alternatively, a way of targeting and killing cancer cells that express high FZD receptors is also being examined. OTSA101 is a humanized monoclonal antibody against FZD10. OTSA101DTPA-90Y (NIH clinical trial number [clinicaltrials.gov] 


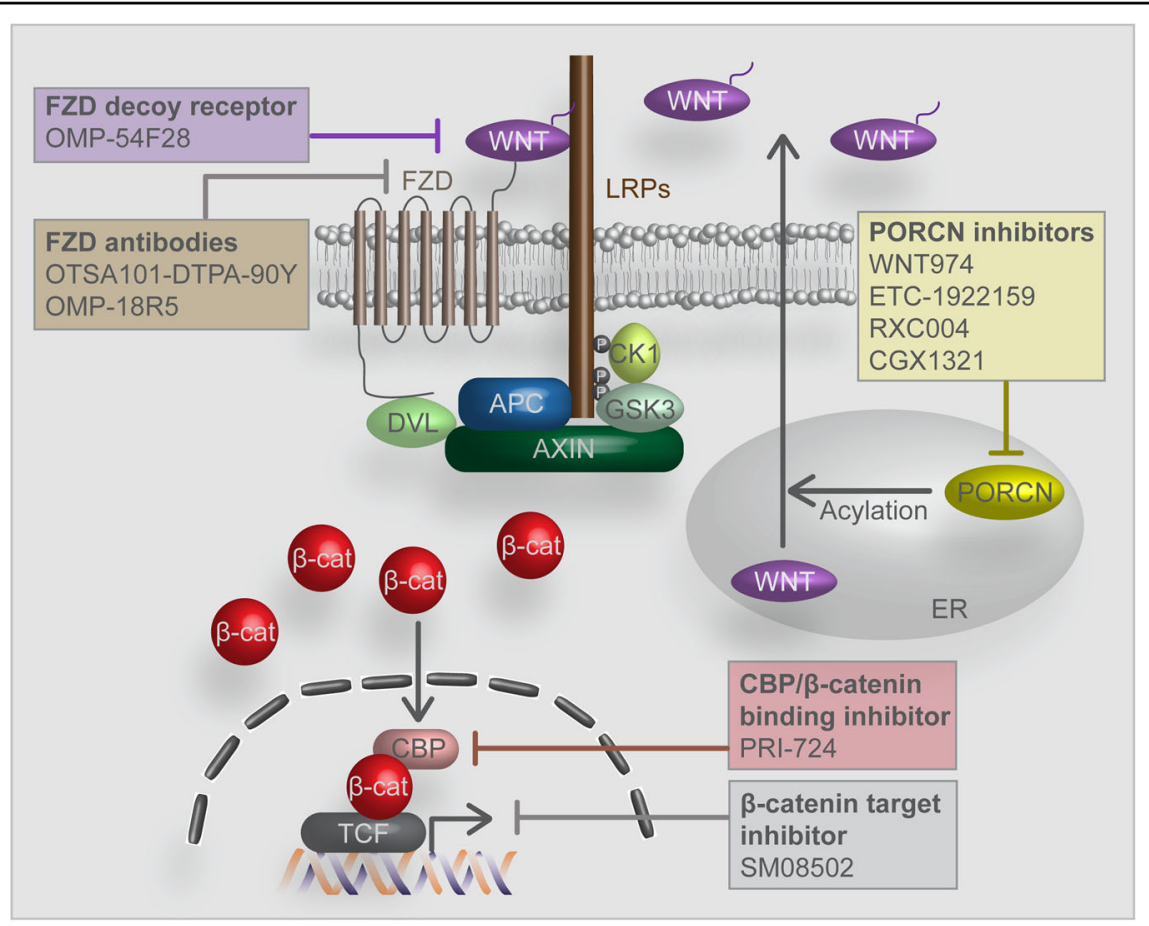

Fig. $1 \mathrm{Wnt} / \beta$-catenin signaling inhibitors in current and past clinical trials (also see Table 1).

NCT01469975) is labeled with a $\beta$-radiation deliveringyttrium Y90 for OSTA101 ${ }^{84}$. OTSA101-DTPA-90Y selectively killed cancer cells highly expressing FZD10. The side effects of vanctumab include tiredness, diarrhea, vomiting, constipation, and abdominal pain. Vantictumab and ipafricept might also cause bone metabolism disorders ${ }^{81,82}$.

(iv) Targeting of LRP degradation and FZD endocytosis Salinomycin, rottlerin, and monensin induce the phosphorylation of LRP6, resulting in the degradation of LRP $^{85-87}$. In addition, niclosamide promotes FZD1 endocytosis, which downregulates WNT3A-stimulated $\beta$-catenin stabilization ${ }^{88}$. However, these reagents do not specifically target cancer-specific molecules, leading to side effects, including itchiness, abdominal pain, vomiting, dizziness, skin rash, and unpleasant taste ${ }^{88,89}$.

Given that the $\beta$-catenin protein destruction complex plays a crucial role in negatively regulating Wnt signaling, the restoration of this protein destruction complex may effectively inhibit Wnt/ $\beta$-signaling. Tankyrase interacts with and degrades AXIN via ubiquitin-mediated proteasomal degradation $^{90-92}$. Tankyrase inhibitors have been developed ${ }^{90,93-95}$. Indeed, Tankyrase inhibitors have been shown to negatively regulate Wnt signaling in $A P C$-mutated cancer cells ${ }^{93-95}$.

(i) Tankyrase inhibitors

Tankyrase inhibitors downregulate $\beta$-catenin stabilization. In preclinical studies, Tankyrase inhibitors, including
XAV939, JW-55, RK-287107, and G007-LK, stabilized AXIN by inhibiting the poly-ADP-ribosylating enzyme Tankyrase ${ }^{90-92}$. However, currently, no clinical trials are being conducted with Tankyrase inhibitors.

(ii) CK1 agonist

Pyrvinium is an FDA-approved anti-helminthic drug. Pyrvinium binds to CK1 family members in vitro and promotes CK1 kinase activity ${ }^{96}$.

$\beta$-Catenin contributes to tumorigenesis via transactivation of Wnt target genes such as CCND1, CD44, $A X I N 2$, and $M Y C^{97-100}$. Thus, approaches inhibiting either $\beta$-catenin transcriptional activity or $\beta$-catenin target genes have been developed as potential therapeutic candidates for Wnt signaling-associated cancers (Table 1).

(i) Inhibitors of $\beta$-catenin transcriptional activity

$\beta$-Catenin/CBP binds to WRE (Wnt-responsive element; 5'-CTTTGA/TA/T-3') and activates target gene transcription $^{101,102}$. PRI-724 (ICG-001; NIH clinical trial numbers: NCT01302405, NCT02413853, NCT01764477, and NCT01606579) inhibits the interaction between CBP and $\beta$-catenin and prevents transcription of Wnt target genes $^{103}$. Moreover, various inhibitors of TCF/LEF and $\beta$-catenin interactions have been identified and evaluated in preclinical settings ${ }^{104}$.

To transactivate Wnt target genes, $\beta$-catenin forms a transcriptional complex with coactivators, including 
BCL9 and PYGO ${ }^{105,106}$, which is inhibited by carnosic acid, compound 22, and SAH-BLC $9^{107,108}$. In addition, Pyrvinium downregulates Wnt transcriptional activity through the degradation of PYGO ${ }^{96}$.

(ii) Inhibitor of Wnt target genes

SM08502 (NIH clinical trial number NCT03355066) is a small molecule that inhibits serine and arginine-rich splicing factor (SRSF) phosphorylation and disrupts spliceosome activity. Upon oral administration, SM08502 was shown to downregulate Wnt signaling-controlled gene expression.

(iii) Proteasomal degradation of $\beta$-catenin

MSAB (methyl 3-[(4-methylphenyl)sulfonyl]amino-benzoate) binds to $\beta$-catenin and facilitates the ubiquitinationmediated proteasomal degradation of $\beta$-catenin ${ }^{108,109}$.

However, since $\beta$-catenin controls various physiological processes, downregulation of the transcriptional activity $\beta$-catenin was shown to induce diarrhea, hypophosphatemia, reversible elevated bilirubin, nausea, fatigue, anorexia, and thrombocytopenia ${ }^{59,110}$.

\section{Additional layers of $W n t / \beta$-catenin signaling activation}

\section{The $\beta$-catenin paradox}

Wnt signaling hyperactivation by mutations in $\beta$-catenin destruction complex components or $\beta$-catenin itself contributes to tumorigenesis. In addition to $A P C$ mutations, $\beta$-catenin can be further activated by additional layers of regulation ${ }^{39,40,111-117}$, which demonstrated the complexity of Wnt signaling deregulation in cancer. Accumulating evidence supports the notion that additional regulatory processes contribute to Wnt signaling hyperactivation in cancer, as demonstrated in the following examples. (a) Mutant APC is still able to downregulate $\beta$-catenin ${ }^{39,40}$. (b) Even in the presence of APC mutations, blockade of WNT ligands triggers apoptosis or growth inhibition ${ }^{40,113,118}$. (c) $\beta$-Catenin fold induction is essential for the activation of $\beta$-catenin target genes ${ }^{119-121}$. (d) Increased AXIN1 by Tankyrase inhibitor suppresses cell proliferation of cancer cells where Wnt/ $\beta$-catenin signaling is genetically hyperactive $\mathrm{e}^{43,90,93,95,122}$. (e) Mutations in RNF43 and ZNRF3 E3 ligases that degrade Wnt receptors contribute to tumor development ${ }^{111,115}$. (f) Ras/ MAPK signaling is also required for Wnt signaling activation $^{112,123}$. These reports suggest that additional layers further enhance Wnt signaling activation in cancer.

\section{The lysosome and Wnt signaling}

The lysosome contains 40 types of hydrolytic enzymes, including cathepsins, which become active under acidic conditions ${ }^{124}$. Lysosomal hydrolytic enzymes mediate the degradation of phagocytosed material and proteolysis of cytosolic proteins through fusion with the multivesicular body (MVB). Luminal acidification of the lysosome is required for lysosomal protein degradation, which is mainly controlled by vacuolar $\mathrm{H}^{+}$transporters in the lysosomal membrane ${ }^{125}$.

Recently, this classical view of lysosomal functions has evolved into new perspectives highlighting the roles of lysosomes in transcriptional regulation and metabolic homeostasis $^{126}$. In human cancer, lysosomal dysfunction is involved in the generation of building blocks, cell proliferation, metastasis, angiogenesis, and tumor suppressor degradation ${ }^{39,127}$.

It has been reported that Wnt signaling is involved in the endocytosis-mediated formation of the LRP signalosome into the MVB ${ }^{123,128}$. GSK3 in the LRP signalosome is sequestered into the MVB, which leads to an increase in the level of cytosolic $\beta$-catenin and inhibition of Wnt signaling ${ }^{129}$. However, decreased GSK3 kinase activity by MVB sequestration lasts approximately $1 \mathrm{~h}^{129,130}$. Moreover, it is unclear how sequestrated APC, GSK3, AXIN, and CK1 in MVB are processed. A recent study showed that clathrin-mediated endocytosis is required for Wnt signaling activation, which is inhibited by $\mathrm{APC}^{131}$. These studies suggest that vesicular acidification and trafficking also play crucial roles in controlling $\mathrm{Wnt} / \beta$-catenin signaling through modulation of the protein destruction complex. Next, we discuss how APC is deregulated for Wnt signaling hyperactivation in cancer cells.

Wnt signaling activation requires v-ATPase (vacuolar $\mathrm{H}^{+}$-ATPase; an electrogenic $\mathrm{H}^{+}$transporter $)^{125,132,133}$. Previous studies imply that in cancer cells, the upregulation of v-ATPase activity might trigger abnormal Wnt/ $\beta$-catenin signaling and contribute to Wnt signalingdependent tumorigenesis. Growing evidence has demonstrated the effect of v-ATPase on various oncogenic processes, including cellular signaling, survival, drug resistance, and metastasis ${ }^{125,134}$. Moreover, the v-ATPase subunits are highly expressed in colorectal, breast, prostate, liver, ovarian, and pancreatic cancer cells ${ }^{135-138}$. The $\mathrm{v}$-ATPase complex is composed of the V1 domain (in the cytosol) and V0 domain (on the membrane) ${ }^{139,140}$. The V1 domain shows reversible disassociation from the V0 domain under physiological conditions, including glucose concentration, starvation of amino acids, and infection of cells by influenza virus ${ }^{141-144}$. Recently, TMEM9 (transmembrane protein 9) was identified as an activator of $\mathrm{v}$ ATPase and is highly expressed in cancer ${ }^{39}$. TMEM9 amplifies Wnt signaling through the v-ATPase-mediated lysosomal protein degradation of $\mathrm{APC}^{39}$. Given that TMEM9 is highly expressed in CRC cells and that Tmem 9 knockout mice are also viable ${ }^{39}$, molecular targeting of TMEM9 may selectively suppress Wnt signaling activity in cancer cells. 


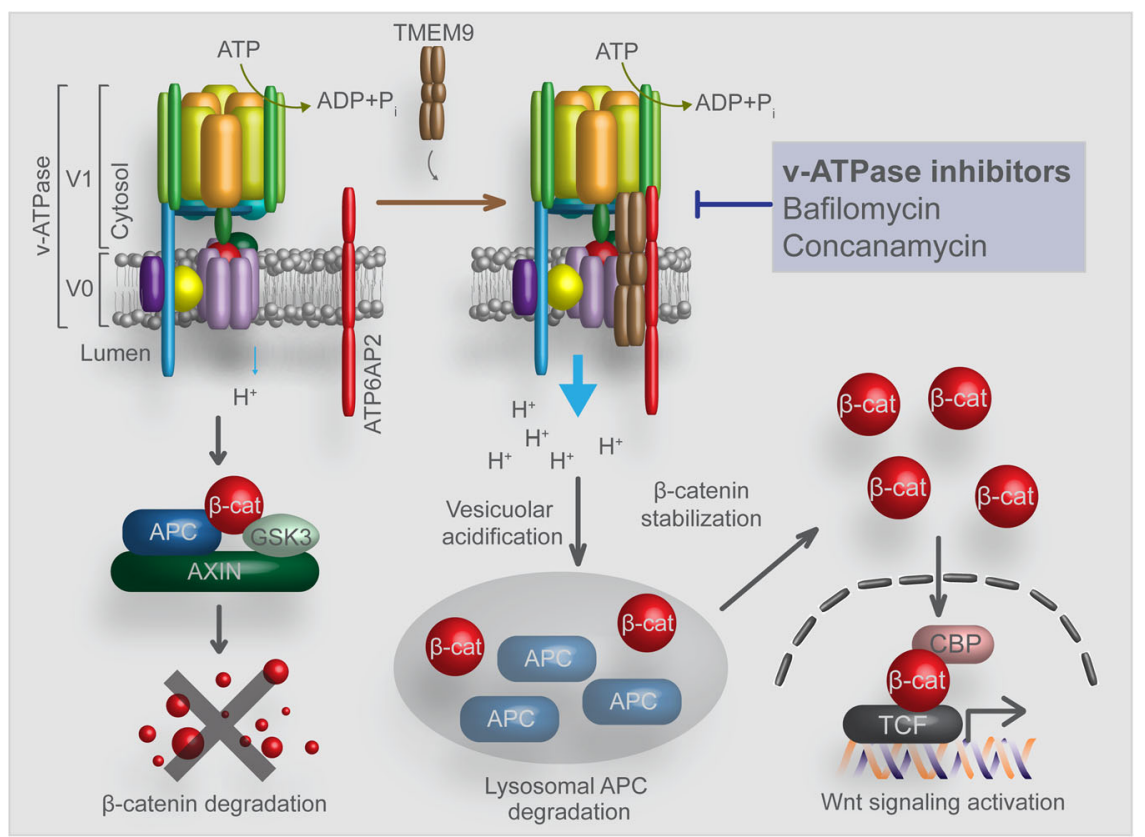

Fig. 2 Inhibition of Wnt/ $\beta$-catenin signaling activity by targeting the TMEM9-v-ATPase axis. TMEM9 expression is highly increased in CRC. As an amplifier of Wnt/ $\beta$-catenin signaling, TMEM9 facilitates the assembly of $\mathrm{v}$-ATPase, resulting in vesicular acidification and subsequent lysosomal degradation of APC. Then, the increased $\beta$-catenin transactivates Wht target genes. The inhibition of TMEM9-V-ATPase-induced vesicular acidification by bafilomycin and concanamycin efficiently inhibits APC lysosomal degradation, which leads to the suppression of Wnt/ $\beta$-catenin gene activation in cancer cells.

\section{Novel therapeutic target: v-ATPase}

Conventional approaches targeting Wnt/ $\beta$-catenin have led to various side effects, as mentioned above. Therefore, cancer-specific Wnt signaling regulators such as vATPase may be attractive molecular targets for Wnt signaling blockade. Chloroquine (CQ) and hydroxychloroquine (HCQ), inhibitors of lysosomes and autophagy, are clinically used for the treatment of diseases such as malaria and rheumatoid arthritis ${ }^{145}$. While the mechanism of action of CQ and HCQ is somewhat unclear, other v-ATPase inhibitors, such as bafilomycin (BAF) and concanamycin (CON), directly bind to and inhibit v-ATPase ${ }^{146,147}$. Compared with CQ and HCQ, BAF and CON showed marked inhibition of Wnt/ $\beta$-catenin signaling in CRC. In addition, BAF and CON displayed an antiproliferative effect in CRC patient-driven xenograft and animal models without toxicity to normal cells and animals ${ }^{39}$. In addition, BAF and CON also strongly inhibit Wnt signaling activity in CRC cells, regardless of $A P C$ mutations. Thus, further research may lead to the development of not only safer but also more potent anti-v-ATPase drugs as cancer-specific Wnt/ $\beta$-catenin inhibitors (Fig. 2).

\section{Conclusion}

Genetic and epigenetic deregulation of Wnt/ $\mathrm{B}$-catenin signaling contributes to human cancer, which has led to the development of extensive approaches targeting Wnt/ $\beta$-catenin signaling as cancer therapies. Nonetheless, the blockade of Wnt signaling impairs tissue homeostasis and regeneration, which needs to be resolved. Recent studies have identified several Wnt signaling regulators whose expression is specific to cancer cells. These cancer-specific regulatory processes of Wnt signaling may be druggable vulnerabilities of Wnt signaling-associated cancer.

\section{Acknowledgements}

This work was supported by grants from the Cancer Prevention and Research Institute of Texas (RP140563 to J.-I.P.), the National Institutes of Health (R01 CA193297-01 to J.-I.P.), the Department of Defense Peer Reviewed Cancer Research Program (W81XWH-15-1-0140 to J.-I.P.), an Institutional Research Grant (MD Anderson Cancer Center to J.-I.P.), SPORE in endometrial cancer (P50 CA83639 to J.I.P.), the Anne Eastland Spears Fellowship in Gastrointestinal Cancer Research (MD Anderson Cancer Center to Y.-S.J.), and the Debbie's

Dream Foundation-American Association for Cancer Research Gastric Cancer Research Fellowship, in memory of Petros Palandjian (19-40-41-Jung to Y.-S.J.).

\section{Author details}

'Department of Experimental Radiation Oncology, Division of Radiation Oncology, The University of Texas MD Anderson Cancer Center, Houston, TX 77030, USA. ${ }^{2}$ Graduate School of Biomedical Sciences, The University of Texas MD Anderson Cancer Center, Houston, TX 77030, USA. ${ }^{3}$ Program in Genetics and Epigenetics, The University of Texas MD Anderson Cancer Center, Houston, TX 77030, USA

Conflict of interest

The authors declare that they have no conflict of interest. 


\section{Publisher's note}

Springer Nature remains neutral with regard to jurisdictional claims in published maps and institutional affiliations.

Received: 15 October 2019 Revised: 20 December 2019 Accepted: 26 December 2019.

Published online: 10 February 2020

\section{References}

1. Acebron, S. P., Karaulanov, E., Berger, B. S., Huang, Y. L. \& Niehrs, C. Mitotic wnt signaling promotes protein stabilization and regulates cell size. Mol. Cell $\mathbf{5 4}$ 663-674 (2014).

2. Atlasi, Y. et al. Wnt signaling regulates the lineage differentiation potential of mouse embryonic stem cells through Tcf3 down-regulation. PLoS Genet. 9, e1003424 (2013)

3. Clevers, H., Loh, K. M. \& Nusse, R. Stem cell signaling. An integral program for tissue renewal and regeneration: Wnt signaling and stem cell control. Science 346, 1248012 (2014)

4. Green, J. L., Inoue, T. \& Sternberg, P. W. Opposing Wnt pathways orient cell polarity during organogenesis. Cell 134, 646-656 (2008).

5. Zhan, T., Rindtorff, N. \& Boutros, M. Wht signaling in cancer. Oncogene $\mathbf{3 6}$, 1461-1473 (2017).

6. Grumolato, L. et al. Canonical and noncanonical Whts use a common mechanism to activate completely unrelated coreceptors. Genes Dev. 24, 2517-2530 (2010).

7. Katoh, M. Canonical and non-canonical WNT signaling in cancer stem cells and their niches: Cellular heterogeneity, omics reprogramming, targeted therapy and tumor plasticity (Review). Int J. Oncol. 51, 1357-1369 (2017).

8. Mirabelli, C. K., Nusse, R., Tuveson, D. A. \& Williams, B. O. Perspectives on the role of Wnt biology in cancer. Sci. Signal. 12, https://doi.org/10.1126/scisignal. aay4494 (2019).

9. Amin, N. \& Vincan, E. The Wht signaling pathways and cell adhesion. Front Biosci. (Landmark Ed.) 17, 784-804 (2012).

10. Li, V. S. et al. Wnt signaling through inhibition of beta-catenin degradation in an intact Axin1 complex. Cell 149, 1245-1256 (2012).

11. MacDonald, B. T., Tamai, K. \& He, X. Wnt/beta-catenin signaling: components, mechanisms, and diseases. Dev. Cell 17, 9-26 (2009).

12. Gao, Z. H., Seeling, J. M., Hill, V., Yochum, A. \& Virshup, D. M. Casein kinase I phosphorylates and destabilizes the beta-catenin degradation complex. Proc. Natl Acad. Sci. USA 99, 1182-1187 (2002).

13. Ha, N. C., Tonozuka, T., Stamos, J. L., Choi, H. J. \& Weis, W. I. Mechanism of phosphorylation-dependent binding of APC to beta-catenin and its role in beta-catenin degradation. Mol. Cell 15, 511-521 (2004).

14. Liu, C. et al. Control of beta-catenin phosphorylation/degradation by a dualkinase mechanism. Cell 108, 837-847 (2002).

15. Stamos, J. L. \& Weis, W. I. The beta-catenin destruction complex. Cold Spring Harb. Perspect. Biol. 5, a007898 (2013).

16. Wu, G., Huang, H., Garcia Abreu, J. \& He, X. Inhibition of GSK3 phosphorylation of beta-catenin via phosphorylated PPPSPXS motifs of Wnt coreceptor LRP6. PLOS ONE 4, e4926 (2009).

17. Schatoff, E. M., Leach, B. I. \& Dow, L. E. Wnt signaling and colorectal cancer. Curr. Colorectal Cancer Rep. 13, 101-110 (2017).

18. White, B. D., Chien, A. J. \& Dawson, D. W. Dysregulation of Wht/beta-catenin signaling in gastrointestinal cancers. Gastroenterology 142, 219-232 (2012).

19. Kwong, L. N. \& Dove, W. F. APC and its modifiers in colon cancer. Adv. Exp. Med. Biol. 656, 85-106 (2009).

20. Rowan, A. J. et al. APC mutations in sporadic colorectal tumors: A mutational "hotspot" and interdependence of the "two hits". Proc. Natl Acad. Sci. USA 97, 3352-3357 (2000).

21. Schell, M. J. et al. A multigene mutation classification of 468 colorectal cancers reveals a prognostic role for APC. Nat. Commun. 7, 11743 (2016).

22. Groden, J. et al. Identification and characterization of the familial adenomatous polyposis coli gene. Cell 66, 589-600 (1991).

23. Kimelman, D. \& Xu, W. beta-catenin destruction complex: insights and questions from a structural perspective. Oncogene 25, 7482-7491 (2006).

24. Xing, $Y$. et al. Crystal structure of a beta-catenin/APC complex reveals a critical role for APC phosphorylation in APC function. Mol. Cell 15, 523-533 (2004).
25. Cadigan, K. M. \& Waterman, M. L. TCF/LEFs and Wnt signaling in the nucleus. Cold Spring Harb. Perspect. Biol. 4, https://doi.org/10.1101/cshperspect. a007906 (2012).

26. Mann, B. et al. Target genes of beta-catenin-T cell-factor/lymphoid-enhancerfactor signaling in human colorectal carcinomas. Proc. Natl Acad. Sci. USA 96, 1603-1608 (1999).

27. Fearon, E. R. \& Vogelstein, B. A genetic model for colorectal tumorigenesis. Cell 61, 759-767 (1990).

28. Hashimoto, K. et al. Cellular context-dependent consequences of Apc mutations on gene regulation and cellular behavior. Proc. Natl Acad. Sci. USA 114, 758-763 (2017).

29. Worm, J., Christensen, C., Gronbaek, K., Tulchinsky, E. \& Guldberg, P. Genetic and epigenetic alterations of the APC gene in malignant melanoma. Oncogene 23, 5215-5226 (2004).

30. Moseley, J. B. et al. Regulated binding of adenomatous polyposis coli protein to actin. J. Biol. Chem. 282, 12661-12668 (2007).

31. Okada, K. et al. Adenomatous polyposis coli protein nucleates actin assembly and synergizes with the formin mDia1. J. Cell Biol. 189, 1087-1096 (2010).

32. Schlessinger, K., McManus, E. J. \& Hall, A. Cdc42 and noncanonical Wnt signal transduction pathways cooperate to promote cell polarity. J. Cell Biol. 178, 355-361 (2007).

33. Watanabe, T. et al. Interaction with IQGAP1 links APC to Rac1, Cdc42, and actin filaments during cell polarization and migration. Dev. Cell 7, 871-883 (2004).

34. Caldwell, C. M. \& Kaplan, K. B. The role of APC in mitosis and in chromosome instability. Adv. Exp. Med. Biol. 656, 51-64 (2009).

35. Hulsken, J., Birchmeier, W., Behrens, J. \& E-cadherin, A. P. C. compete for the interaction with beta-catenin and the cytoskeleton. J. Cell Biol. 127 2061-2069 (1994).

36. Juanes, M. A. et al. Adenomatous polyposis coli nucleates actin assembly to drive cell migration and microtubule-induced focal adhesion turnover. J. Cell Biol. 216, 2859-2875 (2017)

37. Rusan, N. M. et al. CIN: reviewing roles for APC in chromosome instability. J. Cell Biol. 181, 719-726 (2008).

38. Tian, X. et al. E-cadherin/beta-catenin complex and the epithelial barrier. J. Biomed. Biotechnol. 2011, 567305 (2011).

39. Jung, Y. S. et al. TMEM9 promotes intestinal tumorigenesis through vacuolarATPase-activated Wnt/beta-catenin signalling. Nat. Cell Biol. 20, 1421-1433 (2018).

40. Voloshanenko, O. et al. Wnt secretion is required to maintain high levels of Wnt activity in colon cancer cells. Nat. Commun. 4, 2610 (2013).

41. Kishida, M. et al. Axin prevents Wnt-3a-induced accumulation of betacatenin. Oncogene 18, 979-985 (1999).

42. Luo, W. \& Lin, S. C. Axin: a master scaffold for multiple signaling pathways. Neurosignals 13, 99-113 (2004).

43. Mazzoni, S. M. \& Fearon, E. R. AXIN1 and AXIN2 variants in gastrointestinal cancers. Cancer Lett. 355, 1-8 (2014).

44. Salahshor, S. \& Woodgett, J. R. The links between axin and carcinogenesis. J. Clin. Pathol. 58, 225-236 (2005).

45. Haraguchi, K. et al. Ajuba negatively regulates the Wnt signaling pathway by promoting GSK-3beta-mediated phosphorylation of beta-catenin. Oncogene 27, 274-284 (2008).

46. Kim, N. G., Xu, C. \& Gumbiner, B. M. Identification of targets of the Wht pathway destruction complex in addition to beta-catenin. Proc. Natl Acad. Sci. USA 106, 5165-5170 (2009).

47. Dajani, R. et al. Structural basis for recruitment of glycogen synthase kinase 3beta to the axin-APC scaffold complex. EMBO J. 22, 494-501 (2003).

48. Ikeda, S. et al. Axin, a negative regulator of the Wnt signaling pathway, forms a complex with GSK-3beta and beta-catenin and promotes GSK-3betadependent phosphorylation of beta-catenin. EMBO J. 17, 1371-1384 (1998).

49. Kim, S. E. et al. Wnt stabilization of beta-catenin reveals principles for morphogen receptor-scaffold assemblies. Science 340, 867-870 (2013).

50. Willert, K., Shibamoto, S. \& Nusse, R. Wnt-induced dephosphorylation of axin releases beta-catenin from the axin complex. Genes Dev. 13, 1768-1773 (1999).

51. Zeng, X. et al. A dual-kinase mechanism for Wnt co-receptor phosphorylation and activation. Nature 438, 873-877 (2005).

52. MacDonald, B. T. \& He, X. Frizzled and LRP5/6 receptors for Wnt/beta-catenin signaling. Cold Spring Harb. Perspect. Biol. 4, https://doi.org/10.1101/ cshperspect.a007880 (2012). 
53. Zeng, $X$. et al. Initiation of Wnt signaling: control of Wnt coreceptor Lrp6 phosphorylation/activation via frizzled, dishevelled and axin functions. Development 135, 367-375 (2008)

54. He, X., Semenov, M., Tamai, K. \& Zeng, X. LDL receptor-related proteins 5 and 6 in Wnt/beta-catenin signaling: arrows point the way. Development 131, 1663-1677 (2004).

55. Piao, S. et al. Direct inhibition of GSK3beta by the phosphorylated cytoplasmic domain of LRP6 in Wnt/beta-catenin signaling. PLoS ONE 3, e4046 (2008).

56. Stamos, J. L., Chu, M. L., Enos, M. D., Shah, N. \& Weis, W. I. Structural basis of GSK-3 inhibition by N-terminal phosphorylation and by the Wnt receptor LRP6. Elife 3, e01998 (2014).

57. Kishida, S. et al. DIX domains of DVI and axin are necessary for protein interactions and their ability to regulate beta-catenin stability. Mol. Cell Biol. 19, 4414-4422 (1999).

58. Herr, P. \& Basler, K. Porcupine-mediated lipidation is required for Wnt recognition by Wls. Dev. Biol. 361, 392-402 (2012).

59. Zimmerli, D., Hausmann, G., Cantu, C. \& Basler, K. Pharmacological interventions in the Wnt pathway: inhibition of Wnt secretion versus disrupting the protein-protein interfaces of nuclear factors. Br. J. Pharm. 174, 4600-4610 (2017).

60. Liu, J. et al. An integrated TCGA pan-cancer clinical data resource to drive high-quality survival outcome analytics. Cell 173, 400-416 e411 (2018).

61. Grigoryan, T., Wend, P., Klaus, A. \& Birchmeier, W. Deciphering the function of canonical Wnt signals in development and disease: conditional loss- and gain-of-function mutations of beta-catenin in mice. Genes Dev. 22, 2308-2341 (2008).

62. Harada, N. et al. Intestinal polyposis in mice with a dominant stable mutation of the beta-catenin gene. EMBO J. 18, 5931-5942 (1999).

63. Kim, S. \& Jeong, S. Mutation hotspots in the beta-catenin gene: lessons from the human cancer genome databases. Mol. Cells 42, 8-16 (2019).

64. Hrckulak, D., Kolar, M., Strnad, H. \& Korinek, V. TCF/LEF transcription factors: an update from the internet resources. Cancers 8, https://doi.org/10.3390/ cancers8070070 (2016)

65. Orsulic, S. \& Peifer, M. An in vivo structure-function study of armadillo, the beta-catenin homologue, reveals both separate and overlapping regions of the protein required for cell adhesion and for wingless signaling. J. Cell Biol. 134, 1283-1300 (1996).

66. van de Wetering, M. et al. Armadillo coactivates transcription driven by the product of the Drosophila segment polarity gene dTCF. Cell 88, 789-799 (1997).

67. Huber, A. H. \& Weis, W. I. The structure of the beta-catenin/E-cadherin complex and the molecular basis of diverse ligand recognition by betacatenin. Cell 105, 391-402 (2001).

68. Valenta, T., Hausmann, G. \& Basler, K. The many faces and functions of betacatenin. EMBO J. 31, 2714-2736 (2012).

69. Amit, S. et al. Axin-mediated CKI phosphorylation of beta-catenin at Ser 45: a molecular switch for the Wnt pathway. Genes Dev. 16, 1066-1076 (2002).

70. Machin, P. et al. CTNNB1 mutations and beta-catenin expression in endometrial carcinomas. Hum. Pathol. 33, 206-212 (2002).

71. Liu, J. et al. Targeting Wnt-driven cancer through the inhibition of Porcupine by LGK974. Proc. Natl Acad. Sci. USA 110, 20224-20229 (2013).

72. Madan, B. et al. Wnt addiction of genetically defined cancers reversed by PORCN inhibition. Oncogene 35, 2197-2207 (2016).

73. Mullighan, C. G. et al. CREBBP mutations in relapsed acute lymphoblastic leukaemia. Nature 471, 235-239 (2011).

74. Wang, $X$. et al. The development of highly potent inhibitors for porcupine. J. Med Chem. 56, 2700-2704 (2013).

75. Madan, B. et al. Bone loss from Wnt inhibition mitigated by concurrent alendronate therapy. Bone Res 6, 17 (2018).

76. Cheng, C. K. et al. Secreted-frizzled related protein 1 is a transcriptiona repression target of the $t(8 ; 21)$ fusion protein in acute myeloid leukemia. Blood 118, 6638-6648 (2011).

77. Shi, Y., He, B., You, L. \& Jablons, D. M. Roles of secreted frizzled-related proteins in cancer. Acta Pharm. Sin. 28, 1499-1504 (2007).

78. Uren, A. et al. Secreted frizzled-related protein-1 binds directly to Wingless and is a biphasic modulator of Wnt signaling. J. Biol. Chem. 275, 4374-4382 (2000).
79. Gonzalez, D. M. \& Medici, D. Signaling mechanisms of the epithelialmesenchymal transition. Sci. Signal 7, re8 (2014).

80. Lamouille, S., Xu, J. \& Derynck, R. Molecular mechanisms of epithelialmesenchymal transition. Nat. Rev. Mol. Cell Biol. 15, 178-196 (2014).

81. Fischer, M. M. et al. WNT antagonists exhibit unique combinatorial antitumor activity with taxanes by potentiating mitotic cell death. Sci. Adv. 3, e1700090 (2017)

82. Jimeno, A. et al. A first-in-human phase i study of the anticancer stem cell agent ipafricept (OMP-54F28), a decoy receptor for Wnt ligands, in patients with advanced solid tumors. Clin. Cancer Res 23, 7490-7497 (2017).

83. Moore, K. N. et al. A phase 1b dose escalation study of ipafricept (OMP54F28) in combination with paclitaxel and carboplatin in patients with recurrent platinum-sensitive ovarian cancer. Gynecol. Oncol. 154, 294-301 (2019).

84. Giraudet, A. L. et al. A first-in-human study investigating biodistribution, safety and recommended dose of a new radiolabeled MAb targeting FZD10 in metastatic synovial sarcoma patients. BMC Cancer 18, 646 (2018).

85. Lu, D. et al. Salinomycin inhibits Wnt signaling and selectively induces apoptosis in chronic lymphocytic leukemia cells. Proc. Natl Acad. Sci. USA 108 13253-13257 (2011).

86. Tumova, L. et al. Monensin inhibits canonical Wnt signaling in human colorectal cancer cells and suppresses tumor growth in multiple intestina neoplasia mice. Mol. Cancer Ther. 13, 812-822 (2014).

87. Zhu, Y. et al. Rottlerin as a novel chemotherapy agent for adrenocortical carcinoma. Oncotarget 8, 22825-22834 (2017).

88. Li, Y. et al. Multi-targeted therapy of cancer by niclosamide: a new application for an old drug. Cancer Lett. 349, 8-14 (2014).

89. Naujokat, C. \& Steinhart, R. Salinomycin as a drug for targeting human cancer stem cells. J. Biomed. Biotechnol. 2012, 950658 (2012).

90. Huang, S. M. et al. Tankyrase inhibition stabilizes axin and antagonizes Wht signalling. Nature 461, 614-620 (2009).

91. Mariotti, L., Pollock, K. \& Guettler, S. Regulation of Wnt/beta-catenin signalling by tankyrase-dependent poly(ADP-ribosyl)ation and scaffolding. Br. J. Pharm. 174, 4611-4636 (2017)

92. Morrone, S., Cheng, Z., Moon, R. T., Cong, F. \& Xu, W. Crystal structure of a Tankyrase-Axin complex and its implications for Axin turnover and Tankyrase substrate recruitment. Proc. Natl Acad. Sci. USA 109, 1500-1505 (2012).

93. Lau, T. et al. A novel tankyrase small-molecule inhibitor suppresses APC mutation-driven colorectal tumor growth. Cancer Res. 73, 3132-3144 (2013).

94. Menon, M. et al. A novel tankyrase inhibitor, MSC2504877, enhances the effects of clinical CDK4/6 inhibitors. Sci. Rep. 9, 201 (2019).

95. Mizutani, A. et al. RK-287107, a potent and specific tankyrase inhibitor, blocks colorectal cancer cell growth in a preclinical model. Cancer Sci. 109 4003-4014 (2018).

96. Thorne, C. A. et al. Small-molecule inhibition of Wnt signaling through activation of casein kinase 1alpha. Nat. Chem. Biol. 6, 829-836 (2010).

97. He, T. C. et al. Identification of C-MYC as a target of the APC pathway. Science 281, 1509-1512 (1998).

98. Jho, E. H. et al. Wnt/beta-catenin/Tcf signaling induces the transcription of Axin2, a negative regulator of the signaling pathway. Mol. Cell Biol. 22, 1172-1183 (2002).

99. Tetsu, O. \& McCormick, F. Beta-catenin regulates expression of cyclin D1 in colon carcinoma cells. Nature 398, 422-426 (1999).

100. Wielenga, V. J. et al. Expression of CD44 in Apc and Tcf mutant mice implies regulation by the WNT pathway. Am. J. Pathol. 154, 515-523 (1999).

101. Wolf, D., Rodova, M., Miska, E. A., Calvet, J. P. \& Kouzarides, T. Acetylation of beta-catenin by CREB-binding protein (CBP). J. Biol. Chem. 277, 25562-25567 (2002).

102. Yu, W. et al. $\beta$-catenin cooperates with CREB binding protein to promote the growth of tumor cells. Cell Physiol. Biochem 44, 467-478 (2017).

103. Takemaru, K. I. \& Moon, R. T. The transcriptional coactivator CBP interacts with beta-catenin to activate gene expression. J. Cell Biol. 149, 249-254 (2000).

104. Yang, K. et al. The evolving roles of canonical WNT signaling in stem cells and tumorigenesis: implications in targeted cancer therapies. Lab Invest $\mathbf{9 6}$ 116-136 (2016).

105. Cantu, C. et al. A cytoplasmic role of Wnt/beta-catenin transcriptional cofactors Bc|9, Bc|9|, and Pygopus in tooth enamel formation. Sci. Signal. 10 https:/doi.org/10.1126/scisignal.aah4598 (2017).

106. de la Roche, M., Worm, J. \& Bienz, M. The function of BCL9 in Wnt/betacatenin signaling and colorectal cancer cells. BMC Cancer 8, 199 (2008). 
107. Feng, M. et al. Pharmacological inhibition of beta-catenin/BCL9 interaction overcomes resistance to immune checkpoint blockades by modulating Treg cells. Sci. Adv. 5, eaau5240 (2019).

108. Lyou, Y., Habowski, A. N., Chen, G. T. \& Waterman, M. L. Inhibition of nuclear Wnt signalling: challenges of an elusive target for cancer therapy. Br. J. Pharm. 174, 4589-4599 (2017).

109. Hwang, S. Y. et al. Direct targeting of beta-catenin by a small molecule stimulates proteasomal degradation and suppresses oncogenic Wnt/betacatenin signaling. Cell Rep. 16, 28-36 (2016).

110. Cantu, C. et al. The Pygo2-H3K4me2/3 interaction is dispensable for mouse development and Wnt signaling-dependent transcription. Development 140, 2377-2386 (2013).

111. Hao, H. X. et al. ZNRF3 promotes Wnt receptor turnover in an R-spondinsensitive manner. Nature 485, 195-200 (2012).

112. Horst, D. et al. Differential WNT activity in colorectal cancer confers limited tumorigenic potential and is regulated by MAPK signaling. Cancer Res. $\mathbf{7 2}$, 1547-1556 (2012).

113. Jung, Y. S., Jun, S., Lee, S. H., Sharma, A. \& Park, J. I. Wnt2 complements Wnt/ beta-catenin signaling in colorectal cancer. Oncotarget 6, 37257-37268 (2015).

114. Jung, Y. S. et al. Deregulation of CRAD-controlled cytoskeleton initiates mucinous colorectal cancer via beta-catenin. Nat. Cell Biol. 20, 1303-1314 (2018).

115. Koo, B. K. et al. Tumour suppressor RNF43 is a stem-cell E3 ligase that induces endocytosis of Wnt receptors. Nature 488, 665-669 (2012).

116. Jung, $H$. Y. et al. PAF and EZH2 induce Wnt/beta-catenin signaling hyperactivation. Mol. Cell 52, 193-205 (2013).

117. Kim, M. J. et al. PAF-Myc-controlled cell stemness is required for intestinal regeneration and tumorigenesis. Dev. Cell 44, 582-596 e584 (2018).

118. He, B. et al. Blockade of Wnt-1 signaling induces apoptosis in human colorectal cancer cells containing downstream mutations. Oncogene 24, 3054-3058 (2005).

119. Benary, U., Kofahl, B., Hecht, A. \& Wolf, J. Modeling Wnt/beta-catenin target gene expression in APC and Wnt gradients under wild type and mutant conditions. Front Physiol. 4, 21 (2013).

120. Goentoro, L. \& Kirschner, M. W. Evidence that fold-change, and not absolute level, of beta-catenin dictates Wnt signaling. Mol. Cell 36, 872-884 (2009).

121. Herbst, A. et al. Comprehensive analysis of beta-catenin target genes in colorectal carcinoma cell lines with deregulated Wnt/beta-catenin signaling. BMC Genomics 15, 74 (2014).

122. Scarborough, H. A. et al. AZ1366: an inhibitor of tankyrase and the canonical Wnt pathway that limits the persistence of non-small cell lung cancer cells following EGFR inhibition. Clin. Cancer Res. 23, 1531-1541 (2017).

123. Phelps, R. A. et al. A two-step model for colon adenoma initiation and progression caused by APC loss. Cell 137, 623-634 (2009).

124. Johnson, D. E., Ostrowski, P., Jaumouille, V. \& Grinstein, S. The position of lysosomes within the cell determines their luminal pH. J. Cell Biol. 212, 677-692 (2016).

125. Pamarthy, S., Kulshrestha, A., Katara, G. K. \& Beaman, K. D. The curious case of vacuolar ATPase: regulation of signaling pathways. Mol. Cancer 17, 41 (2018).

126. Lawrence, R. E. \& Zoncu, R. The lysosome as a cellular centre for signalling, metabolism and quality control. Nat. Cell Biol. 21, 133-142 (2019).
127. Chi, C. et al. Disruption of lysosome function promotes tumor growth and metastasis in Drosophila. J. Biol. Chem. 285, 21817-21823 (2010).

128. Hagemann, A. I. et al. In vivo analysis of formation and endocytosis of the Wnt/beta-catenin signaling complex in zebrafish embryos. J. Cell Sci. 127, 3970-3982 (2014).

129. Taelman, V. F. et al. Wnt signaling requires sequestration of glycogen synthase kinase 3 inside multivesicular endosomes. Cell 143, 1136-1148 (2010).

130. Ding, V. W., Chen, R. H. \& McCormick, F. Differential regulation of glycogen synthase kinase 3beta by insulin and Wnt signaling. J. Biol. Chem. 275, 32475-32481 (2000).

131. Saito-Diaz, K. et al. APC inhibits ligand-independent Wnt signaling by the clathrin endocytic pathway. Dev. Cell 44, 566-581 e568 (2018).

132. Cruciat, C. M. et al. Requirement of prorenin receptor and vacuolar H+-ATPasemediated acidification for Wnt signaling. Science 327, 459-463 (2010).

133. Hermle, T., Saltukoglu, D., Grunewald, J., Walz, G. \& Simons, M. Regulation of Frizzled-dependent planar polarity signaling by a V-ATPase subunit. Curr. Biol. 20, 1269-1276 (2010).

134. Stransky, L., Cotter, K. \& Forgac, M. The function of V-ATPases in cancer. Physiol. Rev. 96, 1071-1091 (2016).

135. Cotter, K. et al. The a3 isoform of subunit a of the vacuolar ATPase localizes to the plasma membrane of invasive breast tumor cells and is overexpressed in human breast cancer. Oncotarget 7, 46142-46157 (2016).

136. Liu, P., Chen, H., Han, L., Zou, X. \& Shen, W. Expression and role of V1A subunit of V-ATPases in gastric cancer cells. Int J. Clin. Oncol. 20, 725-735 (2015).

137. Ohta, T. et al. Expression of $16 \mathrm{kDa}$ proteolipid of vacuolar-type $\mathrm{H}(+)$-ATPase in human pancreatic cancer. Br. J. Cancer 73, 1511-1517 (1996).

138. Son, S. W. et al. Prognostic significance and function of the vacuolar $H$ +-ATPase subunit V1E1 in esophageal squamous cell carcinoma. Oncotarget 7, 49334-49348 (2016).

139. Inoue, T. \& Forgac, M. Cysteine-mediated cross-linking indicates that subunit $\mathrm{C}$ of the V-ATPase is in close proximity to subunits $\mathrm{E}$ and $\mathrm{G}$ of the $\mathrm{V} 1$ domain and subunit a of the V0 domain. J. Biol. Chem. 280, 27896-27903 (2005).

140. Marshansky, V., Rubinstein, J. L. \& Gruber, G. Eukaryotic V-ATPase: novel structural findings and functional insights. Biochim Biophys. Acta 1837, 857-879 (2014).

141. Kane, P. M. Disassembly and reassembly of the yeast vacuolar $\mathrm{H}(+)$-ATPase in vivo. J. Biol. Chem. 270, 17025-17032 (1995).

142. Kane, P. M. Regulation of V-ATPases by reversible disassembly. FEBS Lett. 469, 137-141 (2000).

143. Rahman, S. et al. Biochemical and biophysical properties of interactions between subunits of the peripheral stalk region of human V-ATPase. PLOS ONE 8, e55704 (2013).

144. Sumner, J. P. et al. Regulation of plasma membrane V-ATPase activity by dissociation of peripheral subunits. J. Biol. Chem. 270, 5649-5653 (1995).

145. Avina-Zubieta, J. A., Galindo-Rodriguez, G., Newman, S., Suarez-Almazor, M. E. \& Russell, A. S. Long-term effectiveness of antimalarial drugs in rheumatic diseases. Ann. Rheum. Dis. 57, 582-587 (1998).

146. Bowman, E. J., Graham, L. A., Stevens, T. H. \& Bowman, B. J. The bafilomycin/ concanamycin binding site in subunit c of the V-ATPases from Neurospora crassa and Saccharomyces cerevisiae. J. Biol. Chem. 279, 33131-33138 (2004).

147. Huss, M. et al. Concanamycin A, the specific inhibitor of V-ATPases, binds to the V(O) subunit C. J. Biol. Chem. 277, 40544-40548 (2002). 